\title{
Interface behavior of tungsten coating on stainless steel by electro spark deposition
}

\author{
Yuangang Wang ${ }^{1, a}$, Honggang $\mathrm{Ma}^{1,}$ Xiaopeng $\mathrm{Li}^{1}$ \\ ${ }^{1}$ Dalian University in Dalian, China
}

\begin{abstract}
A new method of electro spark deposition method was put forward, which was based on the theory of electro spark deposition by changing the polarity in the liquid. Tungsten coating layers was produced on surface of Stainless Steel by electro spark deposition. The micro hardness, microstructure, chemical composition and phases of the coating layer were examined by means of hardness test, scanning electron microscopy (SEM) and energy dispersive spectrometer (EDS) analysis. The results showed that there was tungsten coating in the surface, which was discontinuous. Microhardness of the coating layer was about 3 times more than that of the substrate. The combination between coating layer and substrate was metallurgical bond.
\end{abstract}

\section{Introduction}

Electro spark deposition (ESD) has been widespread concerned for twenty years, which was used to strengthen the surface. At the moment, the electric spark can generate $8000 \sim 25000^{\circ} \mathrm{C}$ temperature when spark discharge. The substrate material depends on the high temperature melting and infiltrating into the workpiece surface. It has been applied to a variety of processing industries, with the characteristic of simple equipment, the use of flexible, large green energy, the thickness of the deposited layer and the advantages of high combination strength. Currently argon is mostly used as the protection gas, and the research tend to study the mechanism on vapor deposition. But the deposition efficiency is low ${ }^{[1-3]}$. And the study for liquid phase deposition is few.

During experiment, electrical discharge spark (EDM) shaping machine was used to deposit tungsten coating on the surface of stainless steel depositing, improved the hardness on the surface of the workpiece. The kerosene that is suggested by the micro-EDM manufacturer is used as a dielectric liquid for the experiments $\left[{ }^{4]}\right.$.To prevent the electrode material flowing with the liquid, circulation pump was closed. It will be beneficial to deposit electrode material. Variety of methods was used to analyze the shape of the workpiece surface, verify the feasibility of this method, and analyze the tungsten coating and the interface of stainless steel organization.
Electro spark deposition in liquid can also achieve the effect of surface strengthening. The hardness elements existing in liquid can react with the substrate material. The new substance can enhance the surface strength.

\section{The experimental materials and methods}

The Sodick 30Ls EDM shaping machine was used in experiment, which the working medium was the kerosene. The pure tungsten, which was selected as electrode, was deposited on a stainless steel substrate in the ESD process experiment. The steel substrate samples (Table 1) was in the form of rectangular plates with the dimensions of $3 \mathrm{~mm} \times 20 \mathrm{~mm} \times 25 \mathrm{~mm}$. The electrode diameter was $\varphi 2 \mathrm{~mm}$. The rotation speed of electrode was $20 \mathrm{rpm} / \mathrm{min}$. Before the experiment, the sandpaper was used to polish the surface and then the acetone was used to clean the steel. The electro spark deposition parameters were shown (Table 2). After deposition, TH763 microhardness tester was used to detect the coating hardness. TH763 microhardness was used to test the hardness of coating microstructure, the value of the loading and time was $100 \mathrm{~g} / \mathrm{N}$ and $5 \mathrm{~s}$ respectively. Produced by the Zeiss EVO-MA-18 scanning electron microscope (SEM) was used to analyze the cross section of the coating layer and morphology. The polarity of electrode was always negative in the experiment.

\footnotetext{
a'Yuanggang Wang: wygang2000@163.com.
} 
Table1. Chemical composition of the matrix material (mass

\begin{tabular}{ccccccc}
\multicolumn{7}{c}{ fraction, \%) } \\
\hline $\mathrm{C}$ & $\mathrm{Si}$ & $\mathrm{Ti}$ & $\mathrm{Cr}$ & $\mathrm{Mn}$ & $\mathrm{Fe}$ & $\mathrm{Ni}$ \\
\hline 3.68 & 0.43 & 0.02 & 18.38 & 1.49 & 68.14 & 7.94 \\
\hline \multicolumn{7}{c}{ Table2. ESD process } \\
\hline Electrical & Pulse & Pulse & Power \\
parameters & $\begin{array}{c}\text { width } \\
\text { interval } \\
\end{array}$ & $\begin{array}{c}\text { ( } \mu \mathrm{s}) \\
(\mu \mathrm{s})\end{array}$ & $\begin{array}{c}\text { Peakply } \\
\text { voltage }(\mathrm{V})\end{array}$ & $\begin{array}{c}\text { current } \\
(\mathrm{A})\end{array}$ \\
\hline Value & 5 & 30 & 120 & 10.1 \\
\hline
\end{tabular}

\section{Experiments and microscopic properties of the coating deposition Mechanism}

\subsection{Coating microhardness}

The hardness distribution curve was shown (Fig 1). The hardest place was on the surface and the value was $1002 \mathrm{HV}$. However, with increasing the distance of the thickness along the coating section, the hardness decreased gradually and then became stabilized. The surface of the coating, which was the highest hardness of the coating, was about 3 times more than that of the matrix. The hardness value of the intermediate region was between the coating and substrate. It can be found that the combination between the transition zone and the substrate was metallurgical bond. This is because of the creation of high temperature, which come to thousands degree that can melt and vaporize the electrode material. These melted and vaporized materials expanded quickly, resulting in exploding and throwing around ${ }^{[5]}$. On the effect of gravity and electric field, these materials contacted the substrate and came together. With the time increased, the substrate surface assembled a lot of electrode material. The deposition coating came into. Partial melted electrode material was vaporized into the matrix in the melt depth.

These results show that because of the present of carbon element, there is carbide in the coating which has higher hardness, which is in the state of grain. The fine grains dispersed in the form of a random distribution. There was a high density of dislocations and residual stress among the fine grains. These factors are the main reason for the deposition layer has high hardness.

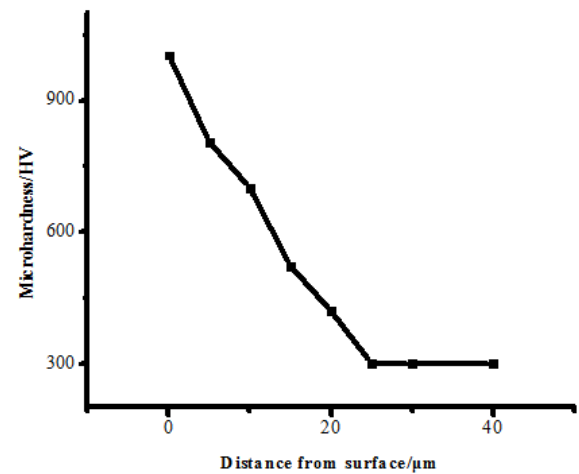

Fig.1 Microhardness distribution of ESD coating

\subsection{Coating surface morphology}

There is a large amount of the coating surface micro pits point, which is due to the deposition process, a large amount of debris belong to the electrode material of discharge pulse generated, is melted into the surface of workpiece (Fig 2). It depends on the explosive force and the gravity. Single point surface morphology of spark deposition was studied. Splashing was fired from the pool center. During deposition process, because the tungsten is refractory material, more substrate material can be melted in the pool in a certain temperature. On the other hand, the coating temperature is higher than that of the substrate, and the closer to the coating surface, the higher the temperature was. This is more benefic for the substrate element to diffuse into the coating layer ${ }^{[6]}$. The substrate elements react with the electrode element, to generate a new phase. With the present of carbon element on the working fluid, the tungsten carbide was generated on the coating layer. This can enhance the hardness of the substrate surface.

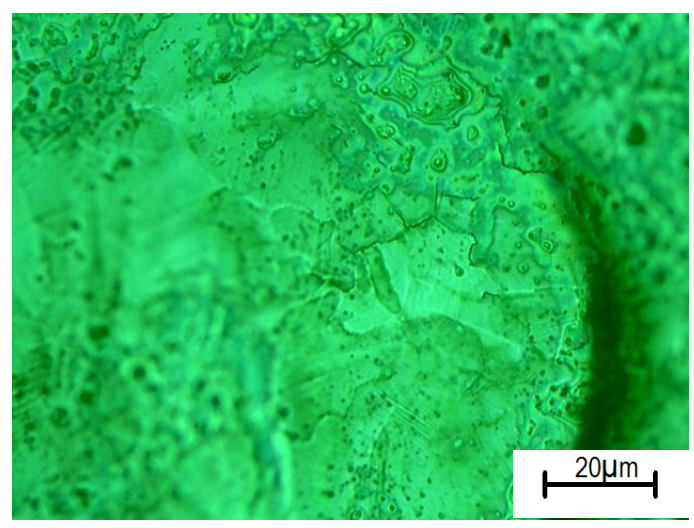

Fig 2. Morphology coating deposited by ESD

\subsection{Coating surface elemental analysis}

It is shown (Fig 3) that the electron microscopy was used to take points on the coating surface. The weight percentage of tungsten and carbon are $63.30 \%$. and $20.85 \%$. According to the above comparison, it shows that there was electrode material deposited in the workpiece surface. But some regions did not have tungsten element. The main reason for this phenomenon is driven by reciprocating movement of the electrode in the flow of the processing liquid, so that splashing and vaporization electrode material movement, there was no spark deposition effect, but it occurred to spark erosion effect ${ }^{[7]}$. In this method, the movement of the electrode feed repeatedly, resulting in nonuniformity in the coating layer, discontinuities, and even in some places there was no coating layer. 


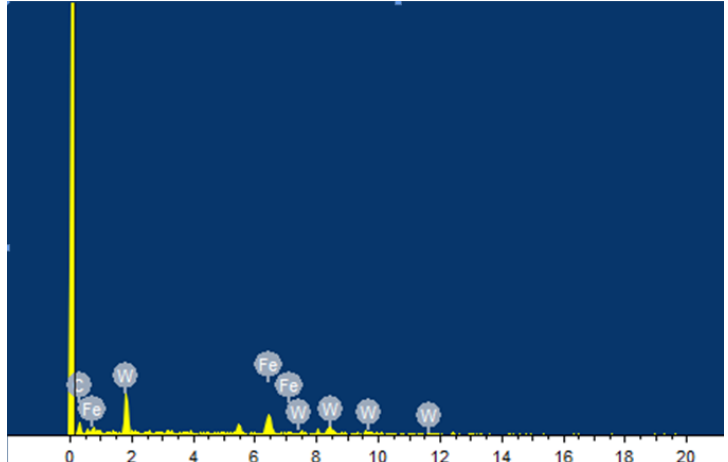

Fig 3. Coating surface elemental analysis

\subsection{Section morphology of the coating}

A cross sectional morphology of the coating was showed (Fig 4), which was cleaned by concentrated nitric acid reagent. It can be seen that the coating thickness is about $5 \mu \mathrm{m}$ and the transition layer region is very small, and then that is the substrate. When the spark surface deposition, the heating speeding was $1 \times 10^{7 \circ} \mathrm{C} / \mathrm{s}$, at the moment the electrode and the workpiece surface is melted; the transition of phase did not occur near the melting area. Because of the temperature increasing, the transformation of the stainless steel phase point increase rapidly ${ }^{[8]}$. But the elevated temperature is limited, the range of phase change widen. Heating phase transformation of austenite was not uniform; the density of defect was high. During electro spark deposition process, a portion of workpiece forms a high vacancy concentration, which produce ultra high temperature (up to $25000^{\circ} \mathrm{C}$ ). Actually rapid heating was a nonequilibrium condition. Nucleation rate was high at this state.

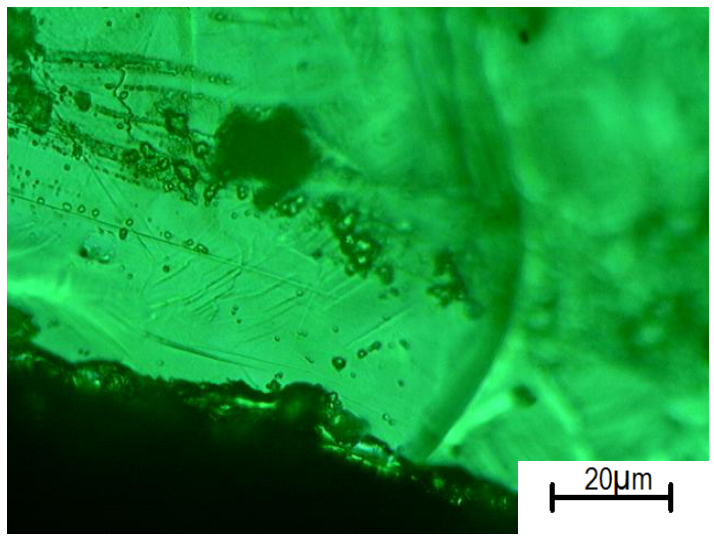

Fig 4. Section morphology of the coating

\section{Conclusion}

1) Electro spark deposition is feasible on a common electro spark machine tools, the thickness of the deposited layer was about $5 \mu \mathrm{m}$;

2) The surface microhardness values of tungsten coating is about 3 times more than that of the substrate, the deposited layer to the hardness of the matrix is decreasing;

3) This method broadens the scope of electro spark deposition, promoting the development of surface hardening technology, solving the problem of liquid electro spark deposition.

\section{Acknowledgements}

The research work was supported by Program for Liaoning Excellent Talents in University. (LJQ2010106)

\section{References}

1. Bulent. E, Atakan. S, Debris and consequences in micro electric discharge machining of microhole $[\mathrm{J}]$. International Journal of machine Tools \& Manufacture, 65, 10 (2013).

2. Alexander.V.R, Orhan.S, The use of bipolar current pulses in electro spark alloying of metal surface[J]. Surface and Coating Technology, 2, 7(2003).

3. Xiaopeng Li, Yuangang Wang, Fuling Zhao, et al. Influence of high frequency pulse on electrode wear in micro-EDM[J]. Defence Technology, 3, 5(2014).

4. Burkoy.A.A, Pyachin.S.A, Investigation of WC Co electrospark coating with various carbon content. journal of Material Engineering and Performance[J]. Journal of Materals Engineering and Performance, 6, 9(2014).

5. Picas.J.A, Xiong.Y, Microstructure and wear resistance of $\mathrm{WC}$ Co by three consolidation processing techniques[J]. International journal of Refracrory Metal and Hard Material, 2, 6 (2009).

6. Wang.J.S, Zhang.Z.J, Interface behavior of WC-Co coating by electro spark deposition[J]. The Chinese journal of Nonferrous Metals, 11, 7 (2014)(in china).

7. Wang.M.W, Pan.R, WC coating on H13 steel surface prepared by electro spark deposition[J]. Transactions of materials and heat treatment, 34, 4(2013) (in china).

8. Wang.R.J, Qian.Y.Y, Liu.J, Structural and interfacial analysis of WC92 Co8 coating deposited on titanium alloy by electrospark deposition [J]. Applied Surface Science, 1, 5(2004)(in china). 